In der Rubrik „Literatur kompakt" werden die wichtigsten Originalarbeiten aus der internationalen Fachliteratur referiert.

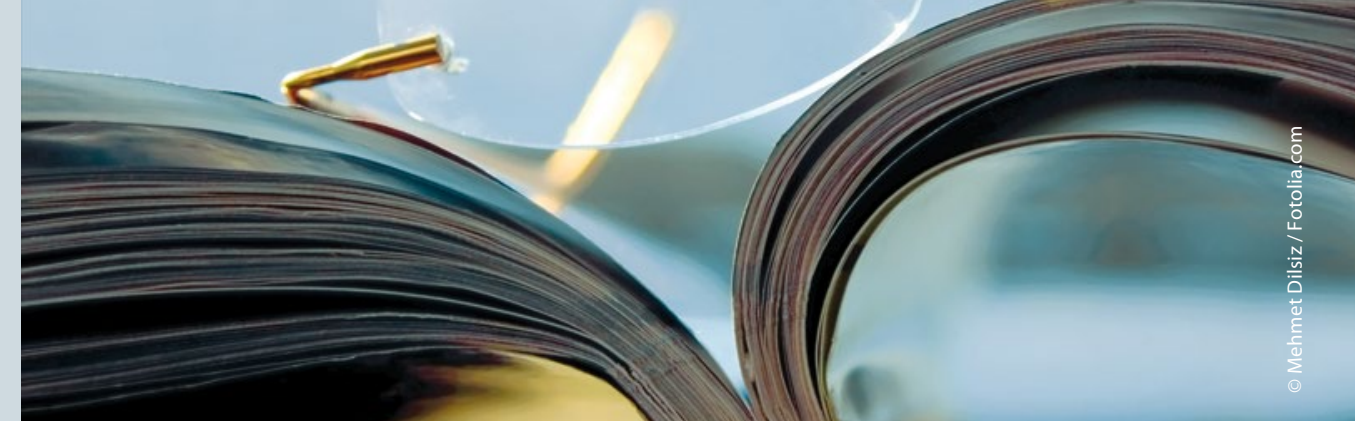

\section{MRT verhindert überflüssige Biopsien}

\section{Mehr als ein Viertel aller Prostatabiopsien lässt sich durch eine kombinierte Magnetresonanztomografie (MRT) vermeiden. Zugleich werden mehr relevante Karzinome erkannt.}

aut den Autoren der randomisiert_kontrollierten PROMIS-Studie gelingt es mit der MRT bei 27 \% aller Männer mit Verdacht auf ein Prostatakarzinom (PCa), überflüssige Prostatabiopsien zu vermeiden. Zugleich soll die MRT $18 \%$ mehr klinisch signifikante PCa aufspüren als eine alleinige per transrektalem Ultraschall (TRUS) geleitete Biopsie.

Für PROMIS konnten englische Urologen um Hashim Ahmed 740 Männer gewinnen, denen aufgrund eines PCaVerdachts erstmals eine Biopsie empfohlen worden war. Alle Männer wurden zunächst per multiparametrischer (mp) MRT untersucht, anschließend boten ihnen die Ärzte eine kombinierte Biopsieprozedur zur Abklärung an. Dabei kamen die Standard-TRUS- und die Template-Biopsie („template prostate mapping“, TPM) zum Einsatz. Letztere wurde als Referenztest verwendet und kann mit einer Sensitivität von $95 \%$ klinisch relevante Karzinome erkennen.

Ahmed et al. untersuchten, ob sich per MRT solche Patienten besser ausschließen ließen, die keine relevanten Tumoren hatten. Dazu beurteilten sie die MRT-Aufnahmen anhand einer FünfPunkte-Skala: Einen Punkt vergaben sie bei einer sehr geringen Wahrscheinlichkeit für ein relevantes Karzinom, zwei Punkte bei einer geringen, drei bei einer $50 \%$-Chance, vier bei hoher und fünf bei sehr hoher Wahrscheinlichkeit. Dieses Punktesystem war zuvor von einer
Konsensusgruppe auf Basis von Registerdaten etabliert worden. Die Hypothese: Patienten mit sehr geringer oder geringer Wahrscheinlichkeit für relevante Tumoren benötigen keine Biopsie. Als klinisch relevant wurden Tumoren mit Gleason-Scores ab $4+3$ sowie einer Tumorkerngröße ab 0,5 mm betrachtet.

Von den Teilnehmern absolvierten 576 die Biopsieprozedur. Bei 408 (71\%) diagnostizierten die Ärzte anhand des TPM einen Tumor, doch nur bei 230 Männern (40\%) fanden sie ein klinisch relevantes Karzinom. Nach den MRT-Daten bestand bei 418 Patienten (73\%) weiterhin Verdacht auf einen relevanten Tumor (3-5 Punkte in der Beurteilung), nicht aber bei den übrigen 158 (27\%; 1-2 Punkte). Von diesen 158 Männern hatten nur 17 einen klinisch signifikanten Tumor, bei den übrigen 141 (89\%) lag die MRT-Beurteilung richtig. Von den 418 Männern mit bestehendem Verdacht hatte rund die Hälfte keinen oder keinen relevanten Tumor. Die multiprozedurale MRT eignet sich wie erwartet vor allem zum Ausschluss von relevanten Tumoren, nicht zu ihrer Diagnose.

Umgekehrt bei der TRUS-Biopsie: Sie spürte bei 124 Männern einen klinisch relevanten Tumor auf, nur 13 davon waren in der TPM-Biopsie negativ. Schlechter war das Resultat bei den 452 Männern ohne Befund: Von ihnen zeigten 119 (26\%) einen relevanten Tumor. Mehr als jedes vierte klinisch relevante Karzi- nom wird bei der Standardbiopsie folglich übersehen. Entsprechend war die Sensitivität mit $93 \%$ bei der mpMRT besonders gut und die Spezifität mit $41 \%$ eher schlecht, dagegen glänzte die TRUS-Biopsie mit einer hohen Spezifität (96\%), dafür aber einer geringen Sensitivität (48\%). Ähnlich verhielt es sich mit den positiven (51 und $90 \%$ ) und negativen prädiktiven Werten (89 und $74 \%$ ) für MRT sowie TRUS-Biopsie.

Fazit: Ahmed et al. simulierten anhand ihrer Studiendaten das naheliegende Verfahren: Zunächst per MRT die Patienten ausschließen, die eine geringe Tumorwahrscheinlichkeit haben, und anschließend bei den übrigen eine Biopsie vornehmen, bevorzugt in den im MRT-Bild auffälligen Bereichen. Damit bleibt $27 \%$ der Männer eine Biopsie erspart, zugleich werden im Optimalfall $18 \%$ mehr relevante Tumoren als mit alleiniger TRUSBiopsie aufgespürt. Mit dieser Kombination entgeht also kaum noch ein klinisch relevanter Tumor, bei $36 \%$ ist die Biopsie aber nach wie vor überflüssig. Immerhin ist dieser Anteil jedoch nur noch halb so hoch wie mit alleiniger TRUS-Biopsie (78\%). Dass die Biopsie nicht harmlos ist, wurde ebenfalls deutlich: Bei acht Männern kam es zur Sepsis, bei 58 zur Harnretention (10\%). Ihre Studie liefere erstmals eine klare Evidenz, wonach das MRT einem wesentlichen Anteil der Männer mit PCa-Verdacht eine Biopsie ohne Nachteil erspart, schussfolgerten Ahmed et al.

Thomas Müller

Ahmed HU et al. Diagnostic accuracy of multiparametric MRI and TRUS biopsy in prostate cancer (PROMIS): a paired validating confirmatory study. Lancet. 2017; doi: 10.1016/S01406736(16)32401-1. 